\title{
Waveguide mode imaging and dispersion analysis with terahertz near-field microscopy
}

\author{
Oleg Mitrofanov, ${ }^{1,2, a)}$ Thomas Tan, ${ }^{1}$ Paul R. Mark, ${ }^{3}$ Bradley Bowden, ${ }^{3}$ and \\ James A. Harrington ${ }^{3}$ \\ ${ }^{1}$ Department of Electronic and Electrical Engineering, University College London, Torrington Place, \\ London WC1E 7JE, United Kingdom \\ ${ }^{2}$ London Center for Nanotechnology, Gordon Street, London WC1H OAH, United Kingdom \\ ${ }^{3}$ Department of Materials Science and Engineering, Rutgers University, 607 Taylor Rd. Piscataway, \\ New Jersey 08854, USA
}

(Received 1 January 2009; accepted 7 April 2009; published online 28 April 2009)

\begin{abstract}
Propagation of terahertz waves in hollow metallic waveguides depends on the waveguide mode. Near-field scanning probe terahertz microscopy is applied to identify the mode structure and composition in dielectric-lined hollow metallic waveguides. Spatial profiles, relative amplitudes, and group velocities of three main waveguide modes are experimentally measured and matched to the $\mathrm{HE}_{11}, \mathrm{HE}_{12}$, and $\mathrm{TE}_{11}$ modes. The combination of near-field microscopy with terahertz time-resolved spectroscopy opens the possibility of waveguide mode characterization in the terahertz band. (C) 2009 American Institute of Physics. [DOI: 10.1063/1.3126053]
\end{abstract}

Terahertz technology moves toward the component integration and it has become essential to develop terahertz waveguides with low transmission loss and small group velocity dispersion. Terahertz time-domain spectroscopy (terahertz-TDS) is potentially ideal for waveguide characterization. However, waveguide transmission spectra measured by terahertz TDS often contain periodic patterns, which are caused by the waveguide mode interference. ${ }^{1}$ The loss and dispersion analysis in this case is difficult without knowledge of the waveguide mode composition.

The mode structure and composition can be determined by imaging the output terahertz wave at the waveguide end. In particular, recently developed collection mode terahertz near-field microscopy has the potential to detect the output wave spatial profile. ${ }^{2}$ The near-field technique is preferred because it gives the mode structure directly, contrary to the far-field imaging. In this letter, we discuss the application of terahertz near-field probe microscopy to determine the mode structure and composition in dielectric-lined hollow cylindrical metallic waveguides. ${ }^{3-5}$ We consider a typical case of multimode propagation of a short terahertz pulse, when the output waveform becomes a superposition of all excited modes. By detecting the spatial distribution of the terahertz pulse field at the waveguide output, we attempt to determine profiles and relative weight of all excited modes experimentally. This knowledge is essential for the loss and dispersion characterization of individual modes and for waveguide design optimization.

Identification of modes in the dielectric-lined hollow cylindrical metallic waveguides is of a particular interest because two modes have low-loss characteristics $(1-3 \mathrm{~dB} / \mathrm{m}$ at $2.5 \mathrm{THz}) .{ }^{4}$ The dominant mode is determined by the waveguide design (specifically, the dielectric layer thickness and the bore diameter) ${ }^{6-9}$ The hybrid $\mathrm{HE}_{11}$ mode is expected to be the lowest loss mode at $\sim 1-3 \mathrm{THz}$ for a waveguide with a relatively thick $(\sim 10 \mu \mathrm{m})$ dielectric layer. However, the $\mathrm{TE}_{01}$ mode with a doughnut shape electric field profile can be

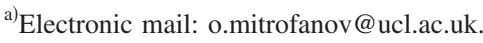

the dominant mode if the dielectric layer is thin $(0-3 \mu \mathrm{m}){ }^{4}$ Mode-specific dispersion characteristics in these waveguides have not been measured yet because several modes are often mixed in transmission experiments. ${ }^{3,10}$

In the experimental setup a horizontally polarized broadband terahertz pulse $(1-2.5 \mathrm{THz})$ is coupled as a slightly diverging wave $\left(\sim 2^{\circ}\right.$ half-angle $)$ into a $133.5 \mathrm{~mm}$ long waveguide with $1.7 \mathrm{~mm}$ bore diameter and the inner coatings of silver $(1 \mu \mathrm{m})$ and polystyrene $(14 \mu \mathrm{m}) .{ }^{11}$ The waveguide output end is scanned with respect to the integrated subwavelength aperture near-field probe. ${ }^{12}$ The probe has an entrance aperture of $50 \mu \mathrm{m}$ and it is positioned within $100 \mu \mathrm{m}$ from the waveguide output end. The resolving power is therefore approximately $100 \mu \mathrm{m}$, independent of the wavelength. Note, that a small tilt of the waveguide during the scan does not affect the wave coupling or propagation in the waveguide because its length is substantially larger $\left(>10^{2}\right)$ than the maximum displacement of the output end. For each waveguide position, the output pulse waveform is measured within a $\sim 70$ ps interval using a standard terahertz spectroscopy setup. It is important to note that the entrance aperture of this near-field probe induces only a minor deformation of the actual pulse waveform because the spectral components of the output pulse lie mostly in the region above the aperture cutoff frequency of $0.86 \mathrm{THz} .^{13}$

The horizontal electric field component of the terahertz pulse waveform measured along the horizontal axis in the plane of the waveguide output end is presented as a spacetime map in Fig. 1(a). The map shows that the input pulse experiences significant changes after propagation through the waveguide. The pulse energy is split into three sections [indicated by arrows in Fig. 1(a)]. The first section (at $t=0$ ) contains most of the pulse energy. The second section, smaller in intensity, is delayed and distributed in a region $t=4-5 \mathrm{ps}$. It shows distinctive phase slips near the halfradius locations. The third section is delayed by about $10 \mathrm{ps}$ and it contains relatively low frequency waves (below $1 \mathrm{THz}$ ). It will be shown later that the first two sections correspond to the $\mathrm{HE}_{11}$ mode and a superposition of the $\mathrm{TE}_{11}$ 


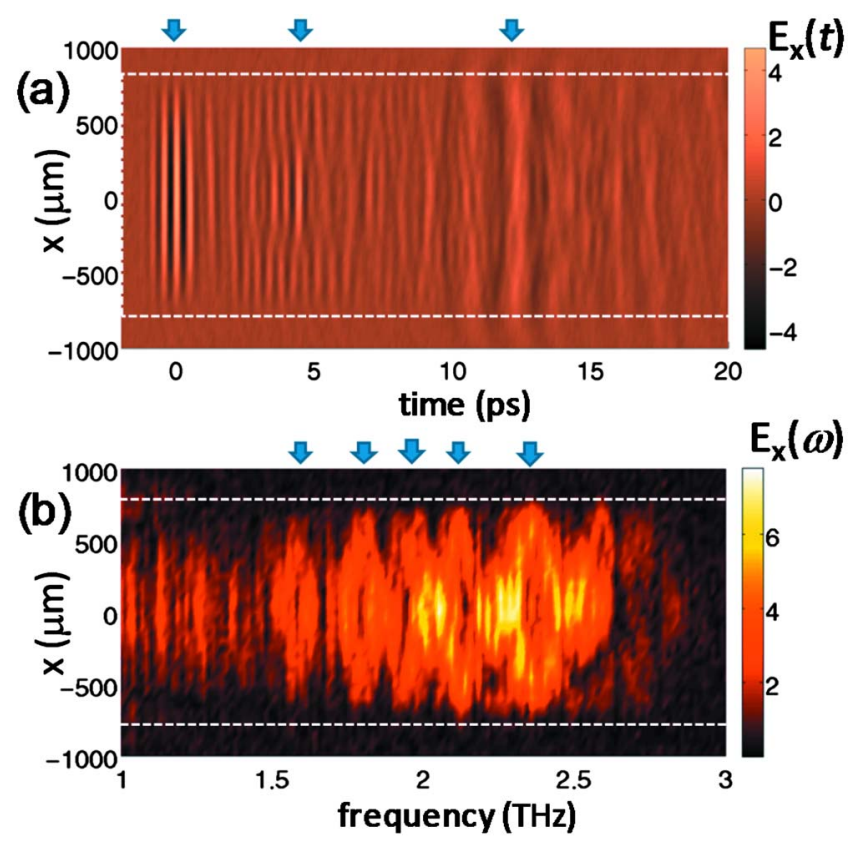

FIG. 1. (Color online) Space-time (a) and space-frequency (b) maps of the horizontal electric field component at the waveguide output measured with the terahertz near-field probe along the horizontal axis. The dashed lines show the inner waveguide wall.

and $\mathrm{HE}_{12}$ modes respectively. Here we focus only on the first two sections, which contain the strongest modes, as they are likely to be of practical importance for terahertz signal transmission applications.

An equivalent map of the electric field spectral amplitude is shown in Fig. 1(b). Distinctive periodic rhombshaped patterns (marked by arrows) and vertical streaks indicate that the incident terahertz pulse couples to more than one waveguide mode, each propagating independently. To uncover the presence of individual modes we apply timedomain windowing to all measured waveforms and analyze the time-domain distribution of their Fourier components. A window function $w(t)$ is chosen to have the full width of $T=1$ ps and smooth edges $\left[w(t)=1 / 2\left\{1+\sin \left[\pi\left(t-t_{w}\right) / T\right]\right\}\right.$, for $\left.t:\left(t_{w}-T\right)<t<\left(t_{w}+T\right)\right]$, and $w(t)=0$ elsewhere]. It ensures that the temporal distribution of the Fourier components above $1 \mathrm{THz}$ can be mapped with a resolution of $1 \mathrm{ps}$.

The distributions in space and time $\left(t_{w}\right)$ for frequencies near the spectral maximum are shown in Fig. 2. The maps clearly display individual modes. The first section has all frequency components of the terahertz pulse confined within a short envelope $(<2 \mathrm{ps})$. It experiences only small broadening and a negative chirp (higher frequencies arrive earlier) as a result of transmission through the waveguide. Absence of any interference patterns indicates that this section contains only one mode split off from other slower traveling modes. The second section is temporally wider with a clear interference pattern.

To identify the modes, spectral amplitude near the pulse maximum $(2.25 \mathrm{THz})$ is plotted as a function of position along the horizontal axis for selected time window delays (Fig. 2 inset). The field amplitude in the first section matches the profile of the radial electric field component of the $\mathrm{HE}_{11}$ mode described by the zeroth order Bessel function. ${ }^{7}$ In the second section the profile matches a superposition of the $\mathrm{TE}_{11}$ and the $\mathrm{HE}_{12}$ modes. Note that the profile changes with

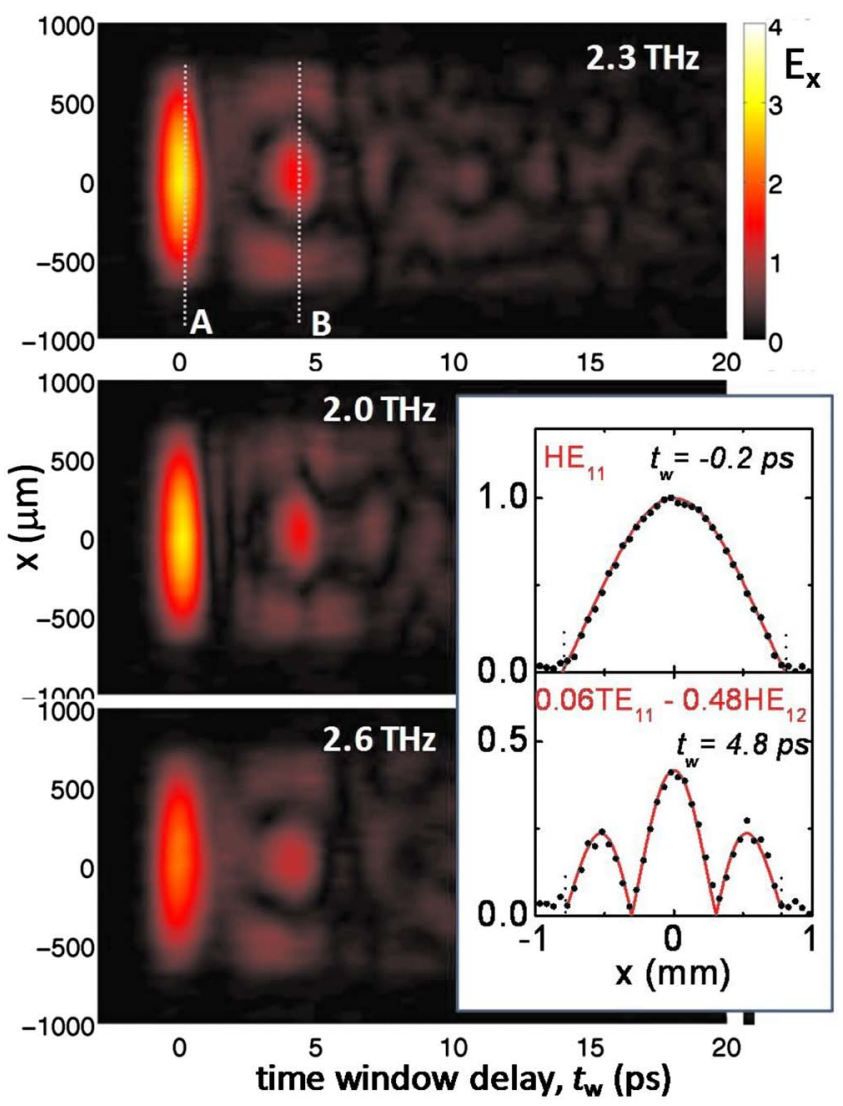

FIG. 2. (Color online) Space-time maps showing temporal distribution of the electric field amplitude for $2.0,2.3$, and $2.6 \mathrm{THz}$ at the waveguide output. Inset: mode profiles (symbols) measured at time window delays A and $\mathrm{B}$ for the peak pulse frequency $(2.25 \mathrm{THz})$. The data are normalized to the peak amplitude of the $\mathrm{HE}_{11}$ mode $\left(t_{w}=-0.2 \mathrm{ps}\right)$. The solid lines show calculated profiles of the horizontal electric field component.

time due to variation of the relative weight of the two interfering modes as expected. This excellent agreement allows identifying the propagating modes despite the temporal overlap of their envelopes. Mode amplitudes normalized to the amplitude of the dominant mode $\mathrm{HE}_{11}$ are listed in Fig. 2. We estimate that the profile maximum of the $\mathrm{HE}_{11}$ mode is approximately 1.7 times larger than the $\mathrm{HE}_{12}$ mode and approximately eight times larger than the $\mathrm{TE}_{11}$ mode.

The origin of the periodic patterns in the spacefrequency map [Fig. 1(b)] becomes clear after performing this mode analysis. The rhomblike patterns are the result of interference of the $\mathrm{HE}_{11}$ mode with the $\mathrm{HE}_{12}$ mode, which displays the phase slips. The pattern periodicity is determined by a relative delay between the interfering modes. For example, five rhomblike patterns within the spectral interval of $1 \mathrm{THz}(1.5-2.5 \mathrm{THz})$ correspond to a relative delay of about 5 ps, which matches the delay between the $\mathrm{HE}_{11}$ and $\mathrm{HE}_{12}$ modes in Fig. 1(a). The delay shows that the group velocity of the $\mathrm{HE}_{12}$ mode is about $1 \%$ lower than that of the $\mathrm{HE}_{11}$ mode. The group velocity for the $\mathrm{HE}_{11}$ mode is $(0.997 \pm 0.001) c$ determined by comparing the pulse propagation time in the waveguide and in free space.

We note that the space-frequency maps in Fig. 2 also give a visual insight into the mode group velocity dispersion. A more temporally confined pulse envelope for the $\mathrm{HE}_{11}$ mode compared to the $\mathrm{HE}_{12}$ and $\mathrm{TE}_{11}$ modes shows that the chromatic dispersion for this mode is the smallest. This experimental result is consistent with the fact that the $\mathrm{HE}_{11}$ 


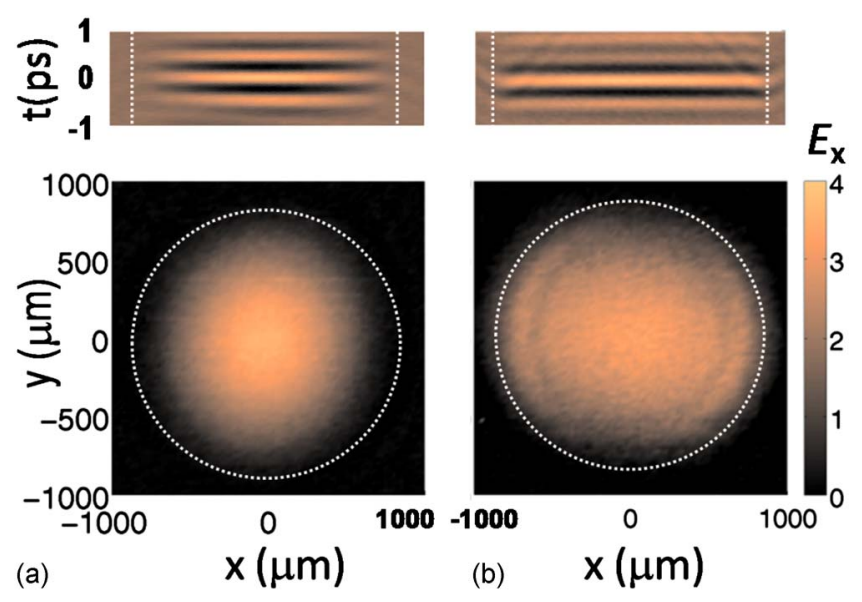

FIG. 3. (Color online) (a) Bottom panel: spatial electric field $\left(E_{x}\right)$ distribution at $t=0$ measured at the output of the waveguide using the terahertz near-field probe; top panel: the corresponding space-time map showing the pulse waveform along the horizontal axis. (b) Similar measurements on a waveguide with the same dimensions however without the dielectric layer.

mode has only a very small fraction of the wave energy propagating near the waveguide walls. Figure 3 (bottom left panel) shows a full two-dimensional scan of the waveguide output end at a fixed time delay $t=0$. The temporal separation of the $\mathrm{HE}_{11}$ mode and the constant phase in the waveguide output plane allow to measure the mode profile directly in time domain. The circular shape of the electric field distribution agrees with the $\mathrm{HE}_{11}$ mode structure. Note that the electric field magnitude gradually decays to zero at the waveguide walls and only a small fraction of the wave travels inside the dielectric layer. Such a profile guarantees small transmission loss and group velocity dispersion as well as good coupling efficiency to and from free space.

This profile is clearly different from the $\mathrm{TE}_{11}$ mode (right panels), measured by the same setup for a waveguide with the same dimensions, however without the dielectric coating. The $\mathrm{TE}_{11}$ mode has an elliptical profile with the electric field terminating abruptly at the side walls. As a result, this mode experiences larger Ohmic losses.

Finally we compare this near-field method to far-field measurements. The spatial variation of the wave front in the waveguide output plane translates into a unique emission pattern. Angular-resolved far-field spectroscopy therefore must show features obtained by the near-field method (assuming that there is no significant presence of evanescent waves at the waveguide output). To verify our near-field results, we measured far-field output waveforms by placing a terahertz photoconductive detector with a hyperhemispheircal lens (1 mm radius) $11 \mathrm{~mm}$ away from the waveguide end. The acceptance aperture of the detector is $\sim 1 \mathrm{~mm}$, corresponding to an angular resolution of $5 \mathrm{deg}$.

The near-field and far-field waveforms are compared in Fig. 4. The far-field waveform measured on the optical axis $\left(\theta=0^{\circ}\right)$ is very similar to the near-field waveform measured at the waveguide center. Some deviation appears at $t=4-5 \mathrm{ps}$, when the $\mathrm{HE}_{12}$ mode is detected by the near-field probe. The reduced signal in the far-field waveform is however expected because the emission in the $\theta=0^{\circ}$ direction is suppressed for the $\mathrm{HE}_{12}$ mode. As the angle $\theta$ increases, the relative intensity of the $\mathrm{HE}_{11}$ mode compared to the $\mathrm{HE}_{12}$

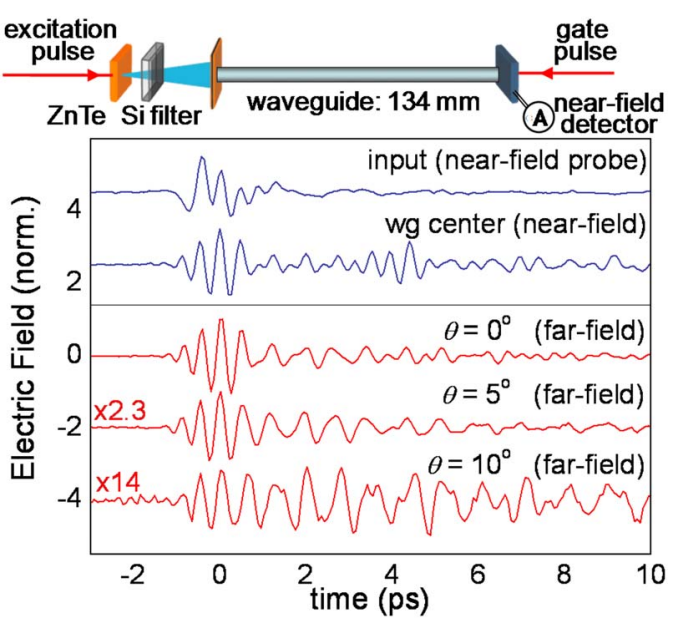

FIG. 4. (Color online) Terahertz pulse waveforms measured in the near-field and the far-field regions. Near-field waveforms are detected by the near-field probe. Angle-resolved waveforms are detected by a photoconductive antenna in the far-field zone. The top waveform shows the input pulse measured by the near-field probe. The schematic diagram shows details of the experimental setup.

decreases and the measured waveforms indeed show this effect qualitatively. Although reconstruction of the mode structure requires measurements of the full angular distribution, Fig. 4 indicates that the results of both methods agree.

In conclusion, this near-field microscopy method gives a valuable tool for research on terahertz waveguides and it will be applied further in studies of wave propagation. Similar to the equivalent method for optical waves, this system allows detection of propagating modes as well as mapping their structure. ${ }^{14}$ We experimentally demonstrate that the dominant linearly polarized mode in the dielectric-lined cylindrical metallic terahertz waveguide is $\mathrm{HE}_{11}$ rather than $\mathrm{TE}_{11}$. The combination of spatially and time-resolved analysis shows that the $\mathrm{HE}_{11}$ mode has the highest group velocity and it experiences relatively small group velocity dispersion in the low-loss transmission region $(2-2.5 \mathrm{THz})$.

${ }^{1}$ G. Gallot, S. P. Jamison, R. W. McGowan, and D. Grischkowsky, J. Opt. Soc. Am. B 17, 851 (2000).

${ }^{2}$ O. Mitrofanov, M. Lee, J. W. P. Hsu, I. Brener, R. Harel, J. Federici, J. D. Wynn, L. N. Pfeiffer, and K. W. West, IEEE J. Sel. Top. Quantum Electron. 7, 600 (2001).

${ }^{3}$ B. Bowden, J. A. Harrington, and O. Mitrofanov, Opt. Lett. 32, 2945 (2007).

${ }^{4}$ B. Bowden, J. A. Harrington, and O. Mitrofanov, Appl. Phys. Lett. 93, 181104 (2008).

${ }^{5}$ Y. Matsuura and E. Takeda, J. Opt. Soc. Am. B 25, 1949 (2008).

${ }^{6}$ C. Dragone, IEEE Trans. Microwave Theory Tech. 28, 704 (1980).

${ }^{7}$ M. Miyagi and S. Kawakami, J. Lightwave Technol. 2, 116 (1984).

${ }^{8}$ C. Themistos, B. M. A. Rahman, M. Rajarajan, K. T. V. Grattan, B. Bowden, and J. A. Harrington, J. Lightwave Technol. 25, 2456 (2007).

${ }^{9} \mathrm{~J}$. A. Harrington, Infrared Fibers and Their Applications (SPIE, Bellingham, WA, 2004).

${ }^{10}$ J. A. Harrington, R. George, P. O. Pedersen, and E. Mueller, Opt. Express 12, 5263 (2004).

${ }^{11}$ B. Bowden, J. A. Harrington, and O. Mitrofanov, J. Appl. Phys. 104, 093110 (2008).

${ }^{12}$ O. Mitrofanov, R. Harel, M. Lee, L. N. Pfeiffer, K. W. West, J. D. Wynn, and J. Federici, Appl. Phys. Lett. 77, 3496 (2000).

${ }^{13}$ O. Mitrofanov, M. Lee, J. W. P. Hsu, L. N. Pfeiffer, K. W. West, J. D. Wynn, and J. Federici, Appl. Phys. Lett. 79, 907 (2001).

${ }^{14}$ J. C. Gates, C. W. J. Hillman, J. C. Baggett, K. Furusawa, T. M. Monro, and W. S. Brocklesby, Opt. Express 12, 847 (2004). 